\title{
Les écoles de dentellières en France et en Belgique des années 1850 aux années 1930
}

Rescuing the Craft. Lace Craft Schools in France and Belgium from the 1850 s to the 1930s

Wie man einem Berufszweig zu Hilfe kommen kann. Schulen für

Spitzenklöpplerinnen in Frankreich und Belgien im Zeitraum zwischen 1850 und 1940

Ayuda para el oficio. Las escuelas de encajeras en Francia y en Bélgica de los años 1850 hasta los años 1930

\section{Stéphane Lembré}

\section{(2) OpenEdition}

Journals

Édition électronique

URL : https://journals.openedition.org/histoire-education/2028

DOI : $10.4000 /$ histoire-education.2028

ISSN : 2102-5452

Éditeur

ENS Éditions

Édition imprimée

Date de publication : 1 juillet 2009

Pagination : 45-70

ISSN : 0221-6280

Référence électronique

Stéphane Lembré, «Les écoles de dentellières en France et en Belgique des années 1850 aux années 1930 », Histoire de l'éducation [En ligne], 123 | 2009, mis en ligne le 01 janvier 2014, consulté le 20 mai 2021. URL : http://journals.openedition.org/histoire-education/2028 ; DOI : https://doi.org/10.4000/ histoire-education.2028 
Au secours du métier

Les écoles de dentellières

en France et en Belgique

des années 1850 aux années 1930

\author{
Stéphane LEMBRÉ
}

Le modèle de la proto-industrialisation a suscité une bibliographie suffisamment abondante ${ }^{1}$ pour qu'il ne soit pas nécessaire d'y revenir à propos de l'activité dentellière. Ici comme ailleurs, "deux âges de la proto-industrie ${ }^{2}$ " peuvent être repérés, et si la production de dentelle, en pleine révolution industrielle, ne déroge pas au processus de mécanisation de la production, la crise qu'elle connait à partir du milieu du XIX ${ }^{e}$ siècle suscite des solutions variées. La situation de cette industrie est amplement commentée à l'occasion d'un regain d'intérêt pour l'art dentellier dans les premières années du XXe siècle $^{3}$.

1 Voir notamment Franklin Mendels, "Des industries rurales à la proto-industrialisation, histoire d'un changement de perspective ", Annales ESC, septembre-octobre 1984, p. 977-1008, et les travaux de Pierre Deyon, par exemple "L'enjeu des discussions autour du concept de proto-industrialisation ", Revue du Nord, janvier-mars 1979, p. 9-18. Plus récemment, René Leboutte (dir.), Proto-industrialisation. Recherches récentes et nouvelles perspectives, Genève, Droz, 1996.

2 Didier Terrier, Les deux âges de la proto-industrie. Les tisserands du Cambrésis et du Saint-Quentinois, 1730-1880, Paris, EHESS, 1996.

3 La liste des écrits consacrés à la dentelle en témoigne : au moins quatre thèses sont soutenues sur ce sujet en moins d'une décennie : Honoré Bayzelon, L'industrie de la dentelle à la main et les tentatives récentes de rénovation, Lyon, Imprimeries réunies, 1906 ; Georges Noé, L'industrie de la dentelle à la main dans le Calvados, Caen, Imprimerie-reliure E. Domin, 1910 ; André Mabille de Poncheville, L'industrie dentellière française spécialement en Flandre, enquête dans la région de Bailleul, Valenciennes, Librairie Giard, 1911 ; B.-J. Poupet, La dentelle d'Alençon, Paris, Arthur Rousseau, 1913. 
Les connaisseurs de la dentelle à la main (qui réunit deux systèmes de travail, aux fuseaux et à l'aiguille) s'accordent globalement sur l'étiologie de la crise : à la mécanisation - synonyme de perte de qualité, de production de masse et de baisse du revenu des dentellières - s'ajoutent la variation trop rapide de la mode, la perte du sens esthétique et la disparition du savoir-faire.

L'industrie dentellière se prête assurément à plusieurs approches ; il ne s'agira pas ici, par exemple, de préciser les rythmes et les traits de cette protoindustrialisation, ni d'interroger l'apprentissage de la technique dentellière, sur lequel s'est penchée Clémence Chevreau ${ }^{4}$. Nous voudrions tenter d'éclairer, sans prétendre nullement à l'exhaustivité, l'un des remèdes préconisés à l'époque face à la crise, à savoir l'enseignement de la dentelle, à partir de cas belges et français. À travers la scolarisation renouvelée de l'apprentissage dentellier, du milieu du XIX ${ }^{\mathrm{e}}$ siècle aux années 1930, il s'agit de décrire l'évolution de la place d'une activité artisanale dans la société, en se fondant sur trois constats. D'abord, l'accent mis sur l'enseignement confirme la perception, face aux conséquences de la mécanisation de la production, d'une solution qualitative aux problèmes engendrés par l'érosion des débouchés et la concurrence accrue entre les centres de production ; "les exigences de la mode " stimulent de ce point de vue les entrepreneurs, comme l'ont montré Philippe Marchand et Didier Terrier ${ }^{5}$. Par ailleurs, l'apprentissage long et contraignant de la dentelle, finalement reconnu par la loi, suppose que le maintien de l'activité réponde à des motivations importantes, notamment d'ordre salarial. Enfin, si l'on a pu critiquer récemment, à tort ou à raison, la tentation propre à certains travaux d'histoire d'une excessive " optique de généalogie du système actuel ${ }^{6}$ " de formation professionnelle, l'industrie dentellière nous semble permettre de contourner cet écueil éventuel : réalité aujourd'hui largement méconnue et cantonnée au rang de curiosité du patrimoine, l'enseignement dentellier n'a jamais été envisagé, au début du XXe siècle, sur un mode systématique, mais a au contraire été proposé et encouragé

4 Clémence Chevreau, "L’Apprentissage de la technique de la dentelle aux fuseaux en Haute-Loire ", Cahiers d'histoire et de philosophie des sciences, hors-série, 2006, p. 309-316.

5 Ph. Marchand, D. Terrier, "Les exigences de la mode et la formation technique des hommes : les écoles d'art et d'industrie à Roubaix et à Tourcoing (fin XIX e siècle) " in Giovanni Luigi Fontana, Gérard Gayot (dir.), Wool: products and markets (13 th $-20^{\text {th }}$ century), Padova, CLEUP, 2004, p. 1195-1203.

6 Claire Lemercier, "Apprentissage " in Alessandro Stanziani (dir.), Dictionnaire historique de l'économiedroit XVIII ${ }^{-} X X^{e}$ siècles, Paris, Libraire générale de droit et de jurisprudence, 2007, p. 23. 
dans des territoires dentelliers anciens par différents acteurs institutionnels, parmi lesquels l'État n'est assurément pas précurseur ${ }^{7}$.

\section{I - Caractéristiques de l'industrie dentellière}

L'évolution du nombre de dentellières, bien que mal connue, suffit à révéler le déclin de l'activité. En un demi-siècle, la France a perdu plus de la moitié de ses dentellières, la saignée étant plus brutale encore en Belgique. L'affaiblissement que l'activité dentellière avait connue à partir de 1788 et durant toute la période révolutionnaire, dont certains centres ne s'étaient remis qu'à grand-peine, tels Lille (16 000 dentellières en 1788, 1600 en 1851) ou Alençon et ses environs, réapparaît avec une acuité accrue au milieu du $\mathrm{XIX}^{\mathrm{e}}$ siècle. À Bruxelles, les affaires des fabricants, en situation délicate en 1847, reprennent en 1849 mais deviennent à nouveau précaires dès la fin des années 1850. De telles oscillations, défavorables aux investissements et à la modernisation du matériel, donc à la pérennisation de la production, caractérisent les décennies suivantes, aussi bien en Belgique qu'en France.

\begin{tabular}{|l|r|r|r|r|}
\hline & $\mathbf{1 8 5 1}^{\mathbf{8}}$ & Vers 1870 & $\mathbf{1 8 9 6}$ & Vers 1906 \\
\hline France & 240000 & - & 127000 & 100000 \\
\hline Belgique & - & 150000 & 47500 & - \\
\hline Normandie & 50000 & - & - & 4 à 5000 \\
\hline Bailleul (Nord) & 8000 & - & - & 1 à 2000 \\
\hline Binche (Belgique) & 150 & - & - & 6 \\
\hline
\end{tabular}

Tableau $\mathrm{n}^{\circ}$ 1. Quelques évaluations du nombre de dentellières à la main ${ }^{9}$

7 Je remercie Jean-François Chanet et Philippe Marchand pour leur relecture suggestive, et leurs éclairages respectifs sur les cas de la Haute-Loire et du Nord.

8 Cette date correspond aux chiffres fournis pour la France par Félix Aubry, industriel dentellier vosgien, qui inventorie la production dentellière française pour l'exposition de Londres. F. Aubry a aussi fait partie des jurys des expositions de Paris en 1849 et 1855. Cf. Georges Vapereau, Dictionnaire universel des contemporains, Paris, Hachette, 1858, p. 73.

9 L'activité est très majoritairement féminine. Pierre Verhaegen recense à peine plus d'une centaine d'hommes parmi les 47000 dentellières belges en 1896 dans La dentelle et la broderie sur tulle, Bruxelles, Office de publicité, 1902, vol 1, chap. 2. 
À partir du milieu du XIX e siècle, mais selon des rythmes propres à chaque centre dentellier, les difficultés s'accumulent pour les fabricants. La dentelle artisanale doit affronter plusieurs obstacles, parmi lesquels la mécanisation croissante de la production, la chute des salaires et les effets de mode. Le marché des produits de la dentelle à la main paraît suffisamment instable pour dissuader fabricants et dentellières de continuer cette activité.

Conséquence de la révolution industrielle, le développement de la dentelle mécanique est rapide. Impulsé notamment à Nottingham dans les premières années du siècle, ce développement est accéléré par l'invention de Jacquard, en 1837, qui introduit des perfectionnements nouveaux dans les dentelles d'imitation. La disparition de la dentelle à la main n'est certes pas immédiate, mais la géographie de la production, basée sur des centres bien identifiés depuis les XVI ${ }^{\mathrm{e}}$-XVII ${ }^{\mathrm{e}}$ siècles, s'en trouve bouleversée. La fortune des tullistes de Calais ou de Caudry, dans le nord de la France, en est une des manifestations les plus spectaculaires ${ }^{10}$. En 1851, il ne reste plus à Valenciennes " que deux vieilles dentellières octogénaires " ${ }^{11}$; la ville a pourtant un passé dentellier glorieux - au point de posséder un type de dentelle éponyme - né au début du XVII ${ }^{\mathrm{e}}$ siècle, rendu possible par l'articulation de la production d'ouvrières expertes, maîtresses dans les institutions religieuses ou caritatives, et de nombreuses façonnières travaillant à domicile ${ }^{12}$. La jeune Belgique, à Ath et Mons, ou Chantilly connaissent le déclin de leur production dentellière tout au long du XIX ${ }^{\mathrm{e}}$ siècle.

Les salaires sont mis en cause par les observateurs du monde dentellier. Le sociologue socialiste Guillaume de Greef, professeur à l'École des sciences sociales de Bruxelles, décrit en des termes saisissants les dentellières belges, " êtres misérables ${ }^{13}$ " dont le sort ne préoccupe personne. La question du salaire est déterminante pour une activité destinée à apporter un revenu supplémentaire au foyer ; dans le département du Calvados, le député Fernand Engerand estime le salaire journalier du millier de dentellières entre dix et quinze sous - « les plus habiles, en travaillant douze ou treize heures, arrivent

10 Voir Michel Caron, Du tulle à la dentelle. Calais 1815/1860, Calais, Le Téméraire, 1997 ; Léonce Bajart, L'industrie des tulles et des dentelles en France. Son établissement dans le Cambrésis, l'essor de Caudry, Lille, Société d'impressions littéraires, industrielles et commerciales, 1953.

11 Fanny Bury Palliser, Histoire de la dentelle, Paris, Firmin-Didot et C ${ }^{\mathrm{ie}}$, 1890, chap. 16.

12 Philippe Guignet, "Les Tribout et leurs ouvrières, symboles et artisans de la brève épopée industrielle des dentelles de Valenciennes ", Revue du Nord, n 6 hors-série, 1995, p. 35-72.

13 Guillaume de Greef, L'ouvrière dentellière en Belgique, Bruxelles, J. Maheu, 1886, p. 6. 
parfois à gagner un franc " ${ }^{14}$. Dès lors, "partout où une industrie accessible aux femmes fait concurrence à la dentelle, celle-ci tend à disparaître " remarque Pierre Verhaegen ${ }^{15}$.

La fragilité des débouchés est inhérente à " l'art industriel le plus subordonné au luxe "16. La nature changeante de la mode interdit à la dentelle à la main d'être autre chose qu'un complément d'activité dans les foyers ruraux. Félix Aubry constate une uniformisation des dentelles qui traduit la concurrence nouvelle entre les centres producteurs de dentelle ${ }^{17}$, et qui rompt avec la diversité des pratiques : les dentelles, en effet, se font soit à l'aiguille, soit au fuseau, sur un métier ou sur un coussin. On en distingue principalement quatre sortes : le point d'Alençon, de France ou de Venise, qui s'exécute tout entier à l'aiguille ; le point de Bruxelles ou d'Angleterre, qui se fait par application - le réseau et les fleurs ne sortent généralement pas de la même main - ; les Malines, faites d'une seule pièce et au fuseau ; enfin, les Valenciennes, qui se fabriquent aussi au fuseau, plus solides que les Malines mais de moindre brillance.

Malgré la concurrence, les dentelles particulièrement précieuses, comme le point d'Angleterre, pratiqué à Bruxelles, la valenciennes d'Ypres, et surtout le point d'Alençon encouragé par Colbert, subsistent. Outre les consécrations littéraires, comme celle que Victor Hugo accorde dans Les Misérables à la dentelle binchoise, lorsque Cosette reçoit de Monsieur Gillenormand une robe en dentelle de Binche dans sa corbeille de noces, le savoir-faire est reconnu lors des temps forts que constituent les expositions, nationales ou universelles ; en 1855, lors de l'Exposition universelle des produits de l'industrie à Paris, la ville de Bayeux reçoit ainsi la grande médaille d'honneur décernée par le jury à l'industrie dentellière. En 1905, la ville de Liège accorde une place toute particulière à la dentelle lors de son Exposition universelle, puisque un Palais lui est dédié.

14 Fernand Engerand, "L'industrie de la dentelle en Normandie ", Revue des Deux Mondes, t. 158, avril 1900, p. 646.

15 Pierre Verhaegen, op. cit., p. 55.

16 G.-Hector Quignon, La dentelle de Chantilly et la question dentellière, Cayeux-sur-Mer, Imprimerie Ollivier, 1905, p. 6.

17 F. Aubry, rapport sur les dentelles, classe XXIV, section I, in Michel Chevalier (dir.), Rapport des membres de la section française du jury international sur l'ensemble de l'exposition universelle, t. 5, Paris, 1862, p. 206. 


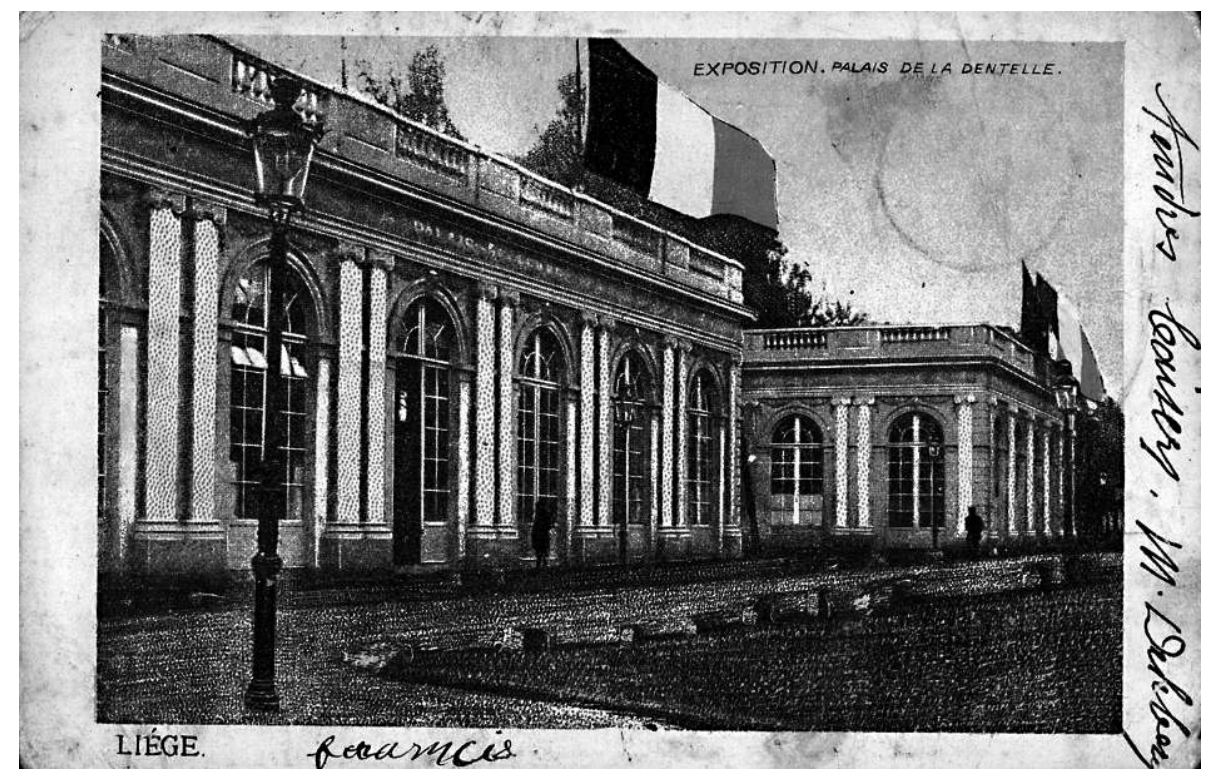

Le Palais de la dentelle à l'Exposition universelle de Liège, en 1905.

(Base Images Laboratoire IRHiS - UMR CNRS 8529 - université Charles-de-Gaulle-Lille 3)

Sur ce fond de difficultés, le regain d'intérêt pour la dentelle à la Belle Époque ne laisse pas d'intriguer. Dès la fin du XIX ${ }^{\mathrm{e}}$ siècle, et surtout dans la première décennie du siècle suivant, l'avenir de la dentelle à la main préoccupe en effet amateurs et pouvoirs publics.

Études de spécialistes, travaux universitaires, expositions, musées et interventions politiques se multiplient. Le musée Galliera, à Paris, destiné à l'art industriel, reconnaît la place de la dentelle parmi les industries d'art en lui consacrant plusieurs expositions ${ }^{18}$, en 1904, 1912 et 1922, mais leurs organisateurs hésitent sur la place que peuvent y prendre les produits mécaniques.

Le renouveau dentellier est stimulé par une prise de conscience et des initiatives fortement territorialisées. À l'instar de Jules Méline déplorant l'exode rural ${ }^{19}$, nombreux sont ceux qui s'inquiètent du manque de vitalité des campagnes. Peu à peu, le parallèle entre exode rural et crise dentellière est établi, et " l'exode urbain " imminent qu'annonce l'emblématique

18 Rossella Froissart-Pezone, "Quand le palais Galliera s'ouvrait aux "ateliers des faubourgs" : le musée d'art industriel de la ville de Paris ", Revue de l'Art, 1997, vol. 116, 1, p. 95-105.

19 Jules Méline, Le retour à la terre et la surproduction industrielle, Paris, Hachette, 3e éd. 1905. 
ministre de l'agriculture de la Troisième République promet une nouvelle vie à la dentelle à la main. La dentelle est produite en effet dans des centres ruraux bien identifiés, dont elle façonne l'identité. En Belgique, les 47571 dentellières dénombrées dans le recensement industriel de 1896 se répartissent dans toutes les provinces, à l'exception de celle de Liège. Elles sont regroupées dans les arrondissements de Thielt, de Dixmude, de Roulers en Flandre occidentale, autour d'Alost et de Termonde en Flandre orientale $^{20}$. En France, des territoires dentelliers subsistent en particulier dans le Nord (Bailleul), en Normandie (à Alençon, autour de Caen et de Bayeux), à Chantilly, dans les Vosges et la Meurthe-et-Moselle (à proximité de Mirecourt, qui compte 25000 dentellières au milieu du siècle), dans la Haute-Saône et la Haute-Loire. À la fin de la monarchie de Juillet, le fabricant de dentelles Théodore Falcon emploie 3500 ouvrières, réparties dans vingt communes autour du Puy. La différence est minime, entre la dentelle du Puy, qui " se mécanise à temps " et celle de l'arrondissement de Bayeux, dont l'effectif s'effondre faute d'avoir adopté la mécanisation : dans ce dernier cas, l'activité dentellière occupe 550 femmes en 1889, alors que 5000 dentellières étaient en activité en $1868^{21}$.

Les périodes de vitalité de la dentelle à la main, dans la seconde moitié du XIX $^{\mathrm{e}}$ siècle, coïncident avec les conjonctures propices aux exportations. Les dentelles proposées en abondance dans les grands magasins parisiens, en effet, ne sont pas les plus luxueuses ; ces dernières sont essentiellement vendues aux hommes d'affaires du nord-est des États-Unis ou à la société aristocratique russe. Ces produits de luxe constituent l'essentiel de la richesse produite par la dentelle à la main en France. Félix Aubry, en 1851, chiffre à soixante-cinq millions de francs environ la valeur de la production annuelle française de dentelle, soit autant que tous les autres pays fabricants réunis.

Aussi la dentelle apparait-elle comme un révélateur social et symbolique de la tension croissante entre deux pôles, l'un formé par le progrès industriel, l'autre par la tradition rurale et artisanale ; d'un côté, la dentelle mécanique, produite en masse, de l'autre la dentelle à la main, œuvre d'art luxueuse. Dans les discours des experts, l'opposition entre la tradition artistique et le progrès mécanique relève de l'évidence, au regard de la crise dentellière :

20 Pierre Verhaegen, La dentelle belge, Bruxelles, Office de publicité, 1912, chap. 2.

21 Denis Woronoff, Histoire de l'industrie en France du XVI $I^{e}$ siècle à nos jours, Paris, Seuil, 1994, p. 218-219 et p. 356 . 
" Notre vingtième siècle donne le curieux spectacle de s'éprendre de l'art le plus délicat, au milieu des réalités les plus brutales de la métallurgie et du machinisme "22. Le "topique de la crise ", appuyé sur un "discours de la décadence ", que repère Anne Rasmussen pour la science à la Belle Époque $^{23}$ se confirme à propos de la dentelle. D'aucuns font le lien entre le déclin du marché de la dentelle et l'évolution du système politique : la cour napoléonienne avait permis à la production de renaître après les événements révolutionnaires nuisibles à la commercialisation de la dentelle comme à la demande, le patronage aristocratique ne stimulant plus la production une fois le système de cour disparu.

Concurrencée et insuffisamment adaptée aux évolutions du marché en amont (production) comme en aval (commercialisation), la dentelle reste une activité pourvoyeuse d'identité sociale et économique pour de nombreux territoires français et belges, au point d'animer un volontarisme scolaire aux enjeux à la fois économiques et sociaux.

\section{II - Le levier scolaire : une formation professionnelle sur mesure}

Sur ces discours abondants se greffent des initiatives scolaires de différents types, qui renouvellent le modèle des anciennes écoles dentellières. La scolarisation de l'apprentissage de la dentelle a tous les traits d'une réaction de survie, qui témoigne d'un volontarisme face à la révolution industrielle venue bouleverser les modes de travail.

Jouer sur la qualité paraît à tous les acteurs du petit monde de la dentelle artisanale la meilleure façon de différencier et préserver leur activité. Néanmoins, compter sur la qualité suppose d'avoir les moyens de la garantir, voire même de l'améliorer, et l'offre d'enseignement dentellier vise donc surtout à atténuer la crise dentellière, à mieux marquer la distance entre la production de masse d'une dentelle grossière et la réalisation d'œuvres luxueuses. Cette logique marque un changement profond du sens de l'institution de formation, dans la mesure où les anciennes écoles dentellières étaient les garantes de la permanence d'un ordre social - non sans fournir

22 G.-Hector Quignon, op. cit., p. 6.

23 Anne Rasmussen, "Critique du progrès, "crise de la science" : débats et représentations du tournant du siècle ", Mil neuf cent, 1996, 1, p. 90. 
un complément de revenu opportun dans les foyers - bien plus que les instruments d'un sauvetage économique ${ }^{24}$. Dans les Flandres belges, le réflexe de fondation d'écoles face à la crise a déjà joué en 1847 dans de nombreux couvents. En Flandre orientale, 300 écoles dentellières s'ajoutent jusqu'en 1851 aux 84 écoles ouvertes avant 1840. Les municipalités agissent avec prudence et font preuve de pragmatisme : ainsi les édiles d'Ath encouragent la création d'un atelier de dentellières en 1846-1847, qui attire peu : en 1847, il y aurait une trentaine d'élèves, et seulement 16 en 1851. Dans un document intitulé "Atelier et école de dentellières ", constatant la disproportion flagrante entre consommation et production, ils évoquent leurs doutes quant à l'avenir de la dentelle dans la cité hennuyère. À l'Exposition provinciale de Mons, en 1851, les produits d'Ath sont jugés des "pièces de dentelles communes ". Pourtant, en 1867, 154 ouvrières sont recensées à Ath, avant un nouveau déclin ${ }^{25}$.

Le même réflexe préside à la démarche d'une habitante belge de Maubeuge, qui demande le patronage de la reine, en février 1841, pour son établissement de dentelles. Le transfert technique est mis en évidence par le sous-préfet, qui confirme qu'elle a apporté quelques années plus tôt " une industrie qui y était inconnue jusque là, celle de la fabrication des dentelles dites point de Binche ". Au même moment, trois négociants tentent de faire revivre l'industrie de la dentelle à Valenciennes ; se heurtant au refus de la municipalité, ils sollicitent l'aide de la commission administrative des Hospices, qui non seulement met à leur disposition une dépendance de l'Hôtel-Dieu, mais établit une école primaire " à la portée des ateliers ", pour que les élèves reçoivent, " en même temps qu'elles se feraient une profession, l'instruction primaire, une éducation morale et religieuse ". Les négociants sont les fabricants de dentelles Marin, de Bruxelles, et Leboulanger, de Paris, associés à " la dame Van Euckout " et réunis dans la société Van Euckout et Cie. Comme leur homologue maubeugeoise, ils requièrent le patronage de la reine " en faveur de leur manufacture pour l'ouverture de laquelle toutes les dispositions préliminaires, tant pour les ateliers que pour l'École qui y sera annexée sont presque terminées "; dans les deux cas, on ne recourt à la bienveillance royale

24 À propos des maîtresses-dentellières valenciennoises du XVIII siècle, Philippe Guignet évoque " une double fonction de production effective et de reproduction du modèle culturel par l'apprentissage ", art. cit., p. 62.

Karl Petit, La dentelle ancienne en Hainaut, Mons, 1982, p. 17. 
qu'une fois le projet développé ${ }^{26}$, et de l'autre côté de la frontière, le réflexe est identique : l'école spéciale ouverte à Mons en 1835 se place de la même façon " sous la protection de la Reine " ${ }^{27}$.

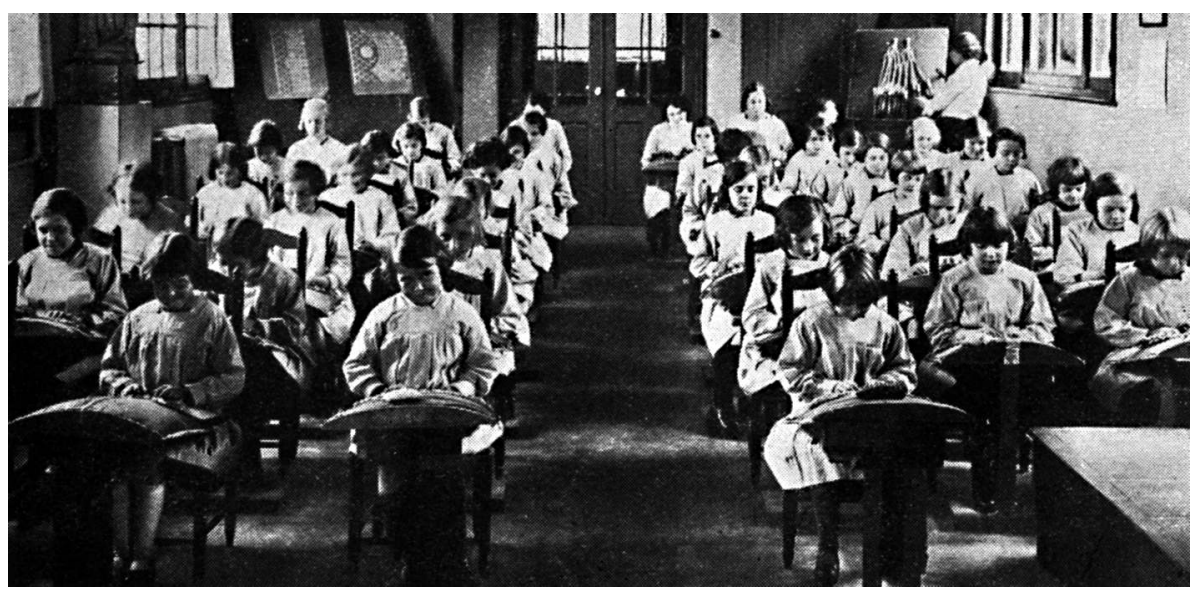

L’École dentellière de Bailleul (Nord) ${ }^{28}$

(Base Images Laboratoire IRHiS - UMR CNRS 8529 - université Charles-de-Gaulle-Lille 3)

Derrière la permanence de l'enseignement dentellier, la forme, l'ampleur et la signification changent. À Bailleul, petite ville de la Flandre française, la première école dentellière, gratuite et destinée à l'éducation des jeunes filles pauvres, a été fondée le 31 juillet 1664 par Anne Swynghedauw. Fidèles à la mémoire de cette pionnière, les Bailleulois célèbrent encore à la fin du XIX ${ }^{\mathrm{e}}$ siècle la Sainte-Anne, en décorant les façades des écoles de dentelle. L'enseignement de la dentelle se maintient ; dans la première moitié du XIX ${ }^{\mathrm{e}}$ siècle, période d'essor de l'industrie dentellière locale, plusieurs écoles privées, soutenues par les fabricants, sont créées à Bailleul et dans les villages environnants. En 1870, ce sont dix écoles qui y assurent l'apprentissage de la main-d'œuvre dentellière selon un modèle faiblement institutionnalisé : le plus souvent, il s'agit d'une maîtresse dentellière qui reçoit à son domicile une vingtaine d'élèves arrivant à l'âge de 4 ou 5 ans,

26 Archives départementales (désormais AD) du Nord, M. 581/13.

27 La Belgique industrielle, Bruxelles, 1836, p. 71, cité par Gustave Casy, "L'école des dentellières de Mons ", Annales du Cercle archéologique de Mons, t. XLV, 1920, p. 38.

28 Extrait de Exposition du progrès social dans les départements libérés et recouvrés, Lille, 1938, p. 54 . 
et s'astreignant en général durant cinq années à un travail de près de dix heures par jour. Les apprenties versent chaque mois un franc, viennent avec leur matériel, et la maîtresse vend ses productions et celles de ses meilleures élèves. En 1888, sous l'effet de la loi scolaire qui impose l'école primaire face à l'apprentissage dentellier, il n'existe plus que quatre écoles, pour une population scolaire de 181 élèves ; en 1891, elles sont 79 apprenties, et une école supplémentaire ferme ses portes. Au tournant du siècle, une seule école subsiste à Bailleul : la dentelle de Valenciennes y est enseignée par Euphrasie Roelandt.

La vie de cette dernière reflète l'évolution de la dentelle : née en 1834, elle apprend la dentelle dès l'âge de cinq ans, et crée en 1856 un ouvroir qu'elle dirige pendant plus d'un demi-siècle. Les cours sont gratuits, puisque l'État alloue vingt francs par élève et par an à Euphrasie Roelandt, et la municipalité bailleuloise lui accorde en juillet 1902 un traitement de 150 francs par an ${ }^{29}$, quoique l'œuvre soit " essentiellement privée et patronnée par Mlle A. Huyghe, marchande de dentelles ${ }^{30}$. D’après la notice rédigée en 1900 pour l'Exposition universelle, le cours consiste en un apprentissage de quatre à cinq années ; les élèves, qui possèdent leur carreau à dentelle, leurs fuseaux et leur fil, travaillent six heures par jour en moyenne. En 1903-1904, un peu moins de trente élèves se rendent à l'école d'Euphrasie Roelandt après la classe primaire et y restent de 11 heures du matin à midi et de 4 heures à 6 heures du soir, ce que le maire, dans une lettre à l'inspecteur d'académie du 28 mai 1903, estime néanmoins insuffisant.

Hors de tout cadre normatif - initialement du moins -, la diversité des formes de l'enseignement de la dentelle est fonction de configurations locales singulières. Partout, à en croire les observateurs, la mère a renoncé à enseigner la dentelle à sa fille, selon le schéma traditionnel de reproduction sociale. Les municipalités et les chambres de commerce, acteurs du territoire, figurent désormais parmi ses principaux promoteurs. Le conseil général du Calvados, en 1884, afin d'encourager les institutrices ou toute maitresse qui prendraient

29 Informations tirées d'Eugène Cortyl, La dentelle à Bailleul, Bailleul, Impr. Ficheroulle-Beheydt, 1903 (extrait du Bulletin du Comité flamand de France, 1903) ; A. Dodanthun, "La fabrication de la dentelle à la main dans le département du Nord ", Mémoires de la Société dunkerquoise pour l'encouragement des sciences, des lettres et des arts, 1905, 41 $1^{\mathrm{e}}$ vol., Dunkerque, Imprimerie dunkerquoise, 1905, p. 5-35 ; André Mabille de Poncheville, op. cit. ; Jacques Lotthé, " La dentelle en Flandre ", Mémoires du Cercle amical des PTT de Lille, section Histoire, t. V, 1984, p. 5-34 ; Mick Fouriscot, La France en dentelles, Paris, Christine Bonneton éd., 1979. 
l'initiative, sur leur temps libre, de donner des leçons de dentelle, vote un crédit de 2000 francs à répartir en primes de 15 francs par élève. En 1900, le crédit est augmenté afin de récompenser les enfants qui s'imposent ce travail supplémentaire ${ }^{31}$. La chambre de commerce d'Alençon décide en 1902 de créer une école dentellière administrée par la chambre, qui reçoit des subventions de l'État, du département et de la commune ${ }^{32}$.

Au Puy, la première école de dentelle est créée en 1838, pour former le goût des dentellières et, déjà, veiller à la qualité. Une deuxième école, dirigée par les béates ${ }^{33}$, ouvre ses portes en 1855 à destination des enfants pauvres de la ville, et accueille une centaine de jeunes filles. Les élus du département, sensibles aux bienfaits et à la prospérité de l'activité, encouragent la création en 1858 d'une école de dessin dentellier, puis en 1862 celle d'une chambre syndicale de la dentelle. Les béates sont toutefois, à la suite de la loi Ferry de 1882 imposant la laïcité de l'enseignement public, très lentement remplacées par des institutrices dénuées de formation dentellière. La main-d'œuvre décroît rapidement, jusqu'à l'intervention de deux dentelliers du Puy, Hippolyte Achard et Pierre Farigoule, qui obtiennent en 1903 l'ouverture d'une section dentellière à l'école pratique de commerce et d'industrie du Puy. La loi de 1903 trouve ici son application en 1910 avec la fondation, au Puy-en-Velay, de l'école de la dentelle au foyer, destinée aux jeunes filles des campagnes alentour. Les cours, gratuits, rencontrent un certain succès, interrompu durablement par la Première Guerre mondiale.

Tous les projets n'aboutissent pas, comme celui qu'a rédigé Arthur Duhem à la demande de la municipalité de Lille et de l'inspecteur général de l'enseignement technique Edmond Labbé en février $1918^{34}$. Les motivations sont ici artistiques et économiques, mais l'auteur lui-même, dans le contexte particulièrement difficile de l'occupation allemande, estime que "la question se pose de savoir s'il est prudent et pratique et surtout s'il est opportun de détourner une partie de cette main-d'œuvre au profit d'une production qui n'est pas de première nécessité ". Son état des lieux identifie les difficultés à

31 Honoré. Bayzelon, op. cit., p. 116.

32 B.-J. Poupet, op. cit., p. 127-129.

33 Corps d'institutrices destinées à donner l'instruction religieuse aux femmes et aux filles des villages et des hameaux et à leur apprendre à lire. Ce mouvement, spécifique à la région du Velay, compte dans les années 1840 plus d'un millier de membres.

34 Arthur Duhem, Projet d'organisation de l'enseignement professionnel de la dentelle, février 1918, dact., Archives municipales de Lille, 4 H 238. 
prévoir, notamment le faible intérêt des ouvrières pour une activité difficile et faiblement rémunératrice. La seule chance de la dentelle serait dès lors de " s'élever dans les régions élevées de l'art et du grand style, inaccessibles à l'imitation mécanique " : dans ce but, une école spéciale de dentelles lui paraît indispensable.

L'intervention législative de l'État n'est pas à l'origine du développement de la formation des dentellières : il s'agit d'accompagner un mouvement né dans les centres producteurs eux-mêmes, et d'abord encouragé par les institutions de proximité. Certes le ministre de l'Instruction publique Victor Duruy prend comme premier exemple du respect des industries locales par son nouvel enseignement spécial les leçons pour le dessin de dentelles au lycée du Puy ${ }^{35}$; l'efficacité au plan local comme dans l'application nationale de la réforme, reste pourtant modeste. Au-delà, l'ensemble de l'intervention de l'État est marquée du sceau de l'ambivalence. Selon les termes de la loi du 5 juillet 1903, "l'enseignement professionnel de la dentelle à la main sera organisé dans les écoles primaires de filles des départements où la fabrication est en usage et dans les écoles normales d'institutrices de ces mêmes départements. Les écoles seront désignées par décret " (article premier) et "il sera créé dans les principaux centres dentelliers des cours et des ateliers de perfectionnement ou des écoles propres à développer l'éducation artistique des ouvrières et des dessinateurs " (article 2). Il s'agit pour l'État de revenir sur les conséquences négatives - pour l'apprentissage de la dentelle - des lois scolaires, notamment la loi Ferry du 28 mars 1882 sur l'enseignement primaire obligatoire. Celle-ci retient les enfants à l'école jusqu'à treize ans, et les inspecteurs, veillant à l'application de la loi, refusent la juxtaposition de l'apprentissage dentellier à l'enseignement primaire. Pour une activité où cet apprentissage requiert jusqu'à une dizaine d'années, et commençait traditionnellement dès la petite enfance, à l'âge de quatre ou cinq ans, le coup est rude et touche à l'avenir même de l'activité. La rentabilité, précaire, disparaît si l'apprentissage débute après la sortie de l'école. 


\begin{tabular}{|c|c|c|c|c|}
\hline Création & Lieu & Forme adoptée & Promoteurs & Remarques \\
\hline 1902 & $\begin{array}{l}\text { Alençon } \\
\text { (Orne) }\end{array}$ & École technique & $\begin{array}{l}\text { Chambre } \\
\text { de commerce }\end{array}$ & \begin{tabular}{|l|}
77 apprenties \\
formées \\
de 1902 à 1912
\end{tabular} \\
\hline 1903 & $\begin{array}{l}\text { Le Puy } \\
\text { (Haute-Loire) }\end{array}$ & $\begin{array}{l}\text { Section } \\
\text { dentellière } \\
\text { à l'EPCI }\end{array}$ & & \\
\hline 1903-1904 & Alençon & $\begin{array}{l}\text { Enseignement } \\
\text { de la dentelle } \\
\text { à l'école } \\
\text { primaire }\end{array}$ & $\begin{array}{l}\text { Application } \\
\text { de la loi du } \\
5 \text { juillet } 1903 \\
\text { et du décret } \\
\text { du } 3 \text { mai } 1904\end{array}$ & $\begin{array}{l}\text { Trois écoles } \\
\text { concernées }\end{array}$ \\
\hline 1904 & \begin{tabular}{|l} 
Bailleul \\
(Nord)
\end{tabular} & $\begin{array}{l}\text { Cours } \\
\text { à l'école } \\
\text { primaire }\end{array}$ & $\begin{array}{l}\text { Arrêté } \\
\text { préfectoral } \\
\text { du } 29 \text { octobre } \\
1904\end{array}$ & $\begin{array}{l}5 \text { h } 30 \text { de cours } \\
\text { par semaine ; } \\
\text { fillettes } \\
\text { de } 7 \text { à } 13 \text { ans }\end{array}$ \\
\hline $\begin{array}{l}1904 \text { ou } \\
1905\end{array}$ & $\begin{array}{l}\text { Le Puy, } \\
\text { Alençon, } \\
\text { Caen }\end{array}$ & $\begin{array}{l}\text { Cours } \\
\text { de dentelle } \\
\text { à l'école normale }\end{array}$ & $\begin{array}{l}\text { Décret du } \\
12 \text { janvier } 1904\end{array}$ & $\begin{array}{l}\text { Un décret du } \\
6 \text { juin } 1905 \\
\text { étend ces } \\
\text { créations aux } \\
\text { écoles normales } \\
\text { d'Arras et } \\
\text { Chambéry }\end{array}$ \\
\hline 1905 & $\begin{array}{l}\text { Verson } \\
\text { (Calvados) }\end{array}$ & $\begin{array}{l}\text { École technique } \\
\text { départementale } \\
\text { de dentelle } \\
\text { au fuseau }\end{array}$ & $\begin{array}{l}\text { Une commission } \\
\text { de surveillance } \\
\text { et une commis- } \\
\text { sion de perfec- } \\
\text { tionnement, } \\
\text { incluant des } \\
\text { fabricants ; } \\
\text { locaux } \\
\text { fournis par la } \\
\text { municipalité }\end{array}$ & $\begin{array}{l}\text { Transfert et } \\
\text { réorganisation } \\
\text { à Caen } \\
\text { en } 1910\end{array}$ \\
\hline 1908 & $\begin{array}{l}\text { Valenciennes } \\
\text { (Nord) }\end{array}$ & $\begin{array}{l}\text { Cours } \\
\text { à l'hôtel de ville }\end{array}$ & $\begin{array}{l}\text { Chambre } \\
\text { de commerce } \\
\text { et municipalité }\end{array}$ & $\begin{array}{l}\text { Expérience } \\
\text { interrompue } \\
\text { en } 1914 \\
\text { par la guerre }\end{array}$ \\
\hline 1910 & $\begin{array}{l}\text { Le Puy } \\
\text { (Haute-Loire) }\end{array}$ & $\begin{array}{l}\text { École } \\
\text { de la dentelle } \\
\text { au foyer }\end{array}$ & & \\
\hline 1920 & \begin{tabular}{|l} 
Bailleul \\
(Nord)
\end{tabular} & École dentellière & $\begin{array}{l}\text { Don de William } \\
\text { Nelson Cromwell }\end{array}$ & $\begin{array}{l}\text { Devient en } 1927 \\
\text { l'École } \\
\text { professionnelle } \\
\text { dentellière, } \\
\text { dans un bâti- } \\
\text { ment dédié }\end{array}$ \\
\hline 1926 & $\begin{array}{l}\text { Valenciennes } \\
\text { (Nord) }\end{array}$ & $\begin{array}{l}\text { Cours aux } \\
\text { écoles } \\
\text { académiques } \\
\text { et à la cité } \\
\text { des cheminots }\end{array}$ & $\begin{array}{l}\text { Municipalité, } \\
\text { Chambre de } \\
\text { commerce et } \\
\text { Compagnie des } \\
\text { chemins de fer } \\
\text { du Nord }\end{array}$ & $\begin{array}{l}80 \text { élèves } \\
\text { en mars } 1926\end{array}$ \\
\hline
\end{tabular}

Tableau $\mathrm{n}^{\circ}$ 2. Quelques créations de cours de dentelle en France au début du XX $\mathrm{XX}^{\mathrm{e}}$ siècle 
Les élus municipaux et les représentants consulaires parviennent à associer le ministère de l'Instruction publique à un effort de modernisation par la voie scolaire dont ils ont au préalable défini les formes. Le projet de loi défendu par le député Fernand Engerand, examiné par le député de la Haute-Loire Louis Vigouroux, désigné comme rapporteur, en 1903, constitue l'aboutissement d'une mobilisation plus large. Léon Lotthé, représentant un canton de Bailleul, avait soulevé le problème devant le conseil général du Nord en 1901. Les diverses expériences scolaires marquaient déjà des accommodements avec la loi de 1882. La loi du 5 juillet 1903, adoptée sans discussion et à l'unanimité par le Sénat et par la Chambre, offre une reconnaissance légale et encourage l'enseignement dentellier. Dès le mois d'octobre 1904, un arrêté préfectoral nomme une institutrice chargée d'enseigner la dentelle aux élèves de l'école publique de filles de Bailleul pendant cinq heures et demie par semaine. En Haute-Loire, vingt-cinq écoles primaires de filles dispensent, grâce à des échantillonneuses ou à des institutrices, un enseignement de la dentelle en avril 1908, d'après l'inspecteur d'académie E. Rayot ; déjà, depuis 1905, la dentelle fait l'objet d'un cours à l'école normale du Puy, et les institutrices sorties depuis de l'établissement sont encouragées à diffuser cet enseignement, à tel point que ce sont une centaine d'écoles qui mettent en valeur la dentelle auprès de "plus de 1500 fillettes ${ }^{36}$, soit environ le tiers des lieux d'accueil potentiels d'une telle formation. Dans cet effort qui, à l'en croire, rencontre des objections nombreuses (par exemple celle de l'inutilité d'un tel enseignement " parce que les fillettes apprennent suffisamment dans leur famille à manier les fuseaux et le carreau "), l'inspecteur d'académie s'appuie sur la collaboration de la Chambre syndicale du Puy et réclame un large soutien. À la logique centralisée de la politique scolaire dans le primaire et le secondaire, selon un modèle nettement amendé par les historiens depuis les années 1990, vient donc s'articuler un modèle territorialisé d'enseignement, dans lequel l'offre et la demande scolaires sont d'abord locales. En témoigne, au demeurant, l'initiative de l'inspecteur d'académie de la Haute-Loire consistant à introduire à partir de 1910 une épreuve de dentelle au certificat d'études primaires (rejoignant, en cela, le cas de la couture). L'inspecteur Rayot est convaincu de la réalité d'une " crise de la dentelle ", et met en avant son engagement personnel pour que l'enseignement

36 Rapport de l'inspecteur d'académie Émile Rayot sur la situation de l'enseignement primaire dans le département de la Haute-Loire pour l'année 1907-1908, Bulletin départemental de l'instruction primaire, Le Puy, juin 1909, p. 220-221. 
dentellier ne soit pas " une mode adoptée pour quelques années, condamnée bientôt à tomber dans l'indifférence ou l'oubli, mais comme une création destinée à durer, à se développer parce qu'elle tient en quelque sorte - par suite de longues traditions - comme au cœur même du pays " ${ }^{37}$. L'inspectrice générale des écoles pratiques de commerce et d'industrie A. Vigneron, membre de la commission chargée d'examiner les mesures à prendre pour l'apprentissage de la dentelle à la main (créée par un arrêté du 29 juillet 1903), mène une enquête sur la dentelle en Italie du Nord. L'école de Burano, qu'elle décrit dans un compte-rendu de voyage publié dans le Bulletin de l'Enseignement technique ${ }^{38}$, admet des jeunes filles âgées au minimum de dix ans, qui doivent justifier d'un enseignement primaire suffisant et d'une bonne santé. Elle compte environ 500 ouvrières et 80 apprenties, avec un chiffre d'affaires de 90000 à 100000 lires qui témoigne de l'insertion de l'école dans l'économie dentellière : les dentellières travaillent exclusivement sur les commandes fournies par l'école.

Les promoteurs de telles initiatives renvoient aux enjeux sociaux du moment. L'enseignement, dans les centres dentelliers, a valeur de remède social au moins autant qu'économique, dans un moment de réflexions et débats pour le développement de l'enseignement ménager dans les écoles de filles ${ }^{39}$. Il s'agit en effet de protéger la cellule familiale, menacée par l'exode rural et l'évolution des valeurs. Bien que de sensibilités diverses, et au prix parfois d'une méconnaissance suffisante pour y déceler " une industrie très saine, se pratiquant dans les beaux jours, en plein air, relativement peu fatigante $"^{40}$, les observateurs trouvent dans la dentelle un remède à la perte du sens moral qu'ils redoutent. "Industrie merveilleusement adaptée aux exigences de la vie des champs et de l'existence féminine ", à en croire Max Turmann ${ }^{41}$, la dentelle serait un facteur de stabilité rurale. La place de la femme dans la société est au cœur de la question dentellière, comme le montre la comparaison de deux études sur le cas belge. Pour Guillaume de Greef, le sort terrible des dentellières mérite d'être érigé en symbole de l'asservissement des travailleurs et témoigne

37 Bulletin départemental de l'enseignement primaire, Le Puy, Imprimerie C. Marguier, 1910, p. 228.

38 A. Vigneron, "La dentelle dans l'Italie du Nord ", Bulletin de l'enseignement technique, publié par l'Association française pour le développement de l'enseignement technique, $\mathrm{n}^{\circ} 2,21$ janvier 1905 , p. 33-43.

39 Voir à ce sujet la thèse de Sandrine Roll, De la ménagère parfaite à la consommatrice engagée. Histoire culturelle de la ménagère nouvelle en France au tournant des XIX ${ }^{e}$ et XX $X^{e}$ siècles, sous la direction de Rebecca Rogers, Université Paris V, 2008.

40 Selon les termes d'Honoré Bayzelon, op. cit., p. 78.

41 Max Turmann, Initiatives féminines, Paris, Victor Lecoffre, 1905, p. 234. 
de "la légitimité de l'ingérence de l'État dans les affaires sociales "42 ; une quinzaine d'années plus tard, Pierre Verhaegen, dans son étude documentée sur la dentelle en Belgique, voit dans le travail de la dentellière à son foyer "la sauvegarde morale des Flandres ": "l'esprit de travail ", le respect des traditions, la méfiance face au monde extérieur au foyer rendent les dentellières " complètement étrangères aux idées de révolte ${ }^{43}$. Le métier possède une signification sociale et politique incontournable dans l'une et l'autre de ces lectures de l'activité dentellière. Raoul Blanchard, dans sa thèse de géographie consacrée à la plaine flamande, perçoit bien l'antagonisme des perceptions lorsqu'il note que l'industrie de la dentelle "diminue malgré l'active campagne menée en sa faveur par le parti conservateur belge, désireux de retenir la femme chez elle, et de l'empêcher d'aller se perdre dans les foules des grandes fabriques ". Mais force est de reconnaitre, par ailleurs, le rôle éminent de la dentelle, " une des rares ressources supplémentaires que peuvent se procurer les femmes " dans les campagnes ${ }^{44}$.

Sans verser dans une taxinomie trop éloignée des réalités sociales, il faut souligner l'équivoque déjà identifiée par Françoise Mayeur : l'enseignement de la dentelle, comme celui de la couture, vise-t-il une formation ménagère ou une formation professionnelle ? En réalité, au tournant des XIX et XX ${ }^{\mathrm{e}}$ siècles, l'enseignement dispensé aux jeunes filles renvoie à des enjeux sociaux multiples ${ }^{45}$ dont, comme l'a justement mis en évidence Michelle Perrot, la relation entre les femmes et les machines atteste de la complexité : "tandis que la machine les introduit dans l'aire publique, on veut obstinément les conserver dans l'espace privé $"^{46}$. Les dentellières, dans cette optique, sont assignées à demeure, rivées à leur carreau, la synthèse de la compétence technique et de la présence féminine au foyer se prêtant parfaitement à la prescription morale en vigueur - non sans laisser ouverte la question de l'importance respective des intérêts économiques et moraux dans l'attachement social à la survie de l'industrie dentellière rurale. La scolarisation de l'apprentissage dentellier, en ces décennies, correspond à

42 G. De Greef, op. cit., p. 11.

43 P. Verhaegen, op. cit., 1912, p. 41.

44 Raoul Blanchard, La Flandre. Étude géographique de la plaine flamande en France, Belgique et Hollande, Lille, L. Danel, 1906, p. 382.

45 Sandrine Roll, op. cit., p. 175-176 notamment.

46 Michelle Perrot, "Femmes et machines au XIX" siècle ", Romantisme, 41, 1983, reproduit dans Les Femmes ou les silences de l'Histoire, Paris, Flammarion, 1998, coll. Champs, p. 182. Voir aussi, dans le même recueil, "Qu'est-ce qu'un métier de femme? ", initialement paru dans Le Mouvement social, 140, 1987, p. 3-8. 
la transition, pratique et symbolique, de la qualité naturelle - dextérité, goût artistique - à la qualification réelle - éventuellement reconnue par un diplôme, mais le plus souvent reléguée à l'arrière-plan de la première. Tout change, grâce à cette mutation, car sortir la dentellière du foyer, c'est aussi la faire apparaître dans le champ d'application de la législation sociale ${ }^{47}$ : la dentelle ne serait plus, ou plus seulement, ce " gain de famine ${ }^{48}$ " qui oblige à produire davantage, au détriment de la qualité, et constituerait l'une des offres locales de la formation ménagère en voie d'institutionnalisation.

Les écoles de dentellières doivent par ailleurs animer les territoires, en retenant les jeunes filles tentées par les places disponibles à la ville. L'acquisition d'un savoir-faire est supposée empêcher les départs de ces jeunes filles qui, pour gagner leur vie, s'engagent comme domestiques. La constitution de comités de patronage de la dentelle à la main est à relier à l'inquiétude suscitée par ce mouvement et à la nostalgie pour la société aristocratique, clientèle privilégiée des fabricants dentelliers. Plusieurs comités de ce type, évoluant entre philanthropie et féminisme, ont vu le jour, comme le Comité de la dentelle en France, qui appuient leur action sur une fondation scolaire ${ }^{49}$.

Ces considérations rejoignent la question plus large de l'intégration nationale. En participant aux œuvres d'enseignement professionnel, les pouvoirs publics atteignent la population des bourgs ruraux, celle-là même dont G. de Greef pointait la résignation face à son sort. La formation professionnelle permet d'insérer le métier individuel dans un collectif au savoir-faire commun. La capacité moralisatrice de l'activité, plus ou moins abandonnée par la sphère privée, est en quelque sorte récupérée dans une perspective nouvelle : ceci rappelle, assurément, que dans les dernières décennies du XIX $\mathrm{X}^{\mathrm{e}}$ siècle, les républicains au gouvernement en France, et les catholiques en Belgique (arrivés au pouvoir en 1884), s'intéressent à l'enseignement professionnel, vu à travers le prisme d'une figure idéale, en l'occurrence celle de la femme au foyer, capable d'apporter un complément de revenu au ménage, garante de la moralité, porteuse en outre d'une compétence artistique. L'on souscrit ainsi à l'analyse selon laquelle " depuis le XIX ${ }^{e}$ siècle, ce ne serait pas tant le travail professionnel et rémunéré de la femme qui serait condamné, symboliquement,

47 Sylvie Schweitzer, Les femmes ont toujours travaillé. Une histoire du travail des femmes aux XIX et XXe siècles, Paris, Odile Jacob, 2002, p. 133 et suiv.

48 R. Blanchard, op. cit., p. 383.

49 André Mabille de Poncheville, op. cit., p. 24-25. 
que ce travail en dehors du contexte familial et sans la reconnaissance du rôle premier et "naturel" de la femme, d'assurer par son travail ménager, l'entretien de la structure familiale et le renouvellement de la force de travail. Sur la base de ce principe, tout l'enseignement professionnel et technique féminin concourt à la formation d'une productrice définie, initialement, comme une productrice ménagère " ${ }^{50}$. L'apprentissage de la dentelle participe pleinement de cette logique, si différente de l'enseignement conventuel ou privé en vigueur, bon an mal an, jusqu'au milieu du XIX ${ }^{\mathrm{e}}$ siècle.

\section{III - Les limites de l'institutionnalisation de l'enseignement de la dentelle}

Malgré le volontarisme de ses promoteurs et le dynamisme institutionnel créé, l'enseignement de la dentelle ne dispose que d'une faible efficacité. La mobilisation scolaire affecte une population réduite, et l'encouragement législatif n’entrave guère la réalité sociale et économique.

Les écoles n'apportent pas les satisfactions escomptées. L'inspecteur d'académie Rayot signale les difficultés de l'organisation de l'enseignement de la dentelle : " bien souvent, les familles refusent d'acheter le petit matériel nécessaire "; de plus, les institutrices, de son propre aveu, manquent d'habileté manuelle et " souvent, à cet égard, leurs élèves en savent plus qu'elles " ${ }^{51}$. Les rapports des années suivantes sont silencieux à propos de la dentelle en HauteLoire, mais le succès local de cet enseignement n'est pas pour autant à exclure. Quant à l'école de Verson, elle est transférée à Caen et réorganisée, en 1910, sous l'impulsion de la Chambre de commerce, mais l'effort scolaire ne suffit pas : " la création de nouveaux débouchés et la réorganisation de l'apprentissage doivent être concomitants, si l'on veut tenter sérieusement de relever l'industrie dentellière ", commente Georges Noé ${ }^{52}$. En installant l'établissement à Caen, par le passage d'un centre rural à la ville, ses promoteurs modifient le sens de l'institution : les élèves potentielles sont plus nombreuses, mais la décision ne tient pas compte de la faible capacité de la dentelle à fidéliser sa main-d'œuvre lorsque s'offrent d'autres emplois exigeant un savoir-faire moindre. Parmi les

50 Dominique Grootaers (dir.), Histoire de l'enseignement en Belgique, Bruxelles, CRISP, 1998, p. 417-418.

51 Émile Rayot, op. cit., 1910, p. 229-230.

52 Georges Noé, op. cit., p. 169. 
77 apprenties formées à l'école dirigée par la chambre de commerce d'Alençon de 1902 à 1912, un quart seulement continue la fabrication. Si le travail autour des tissus, premier secteur d'emploi des femmes, demeure, la baisse de la part du travail à domicile modifie son économie interne. L'ambiguité de l'activité dentellière, "travail d'appoint, travail de misère "53, n'est pas dissipée par la scolarisation de l'apprentissage.

C'est à une recherche systématique de la qualité, principale réponse à la concurrence des dentelles mécaniques et des produits belges, que renvoie cette scolarisation. Devant la commission d'enquête sur la situation des ouvriers et des industries d'art, en 1883, des fabricants de dentelles d'Alençon, de Bailleul, de Bayeux et du Puy présentent leurs desiderata ${ }^{54}$. Des divergences apparaissent : Huignard, fabricant à Alençon, se soucie de la formation des dentellières et ne craint guère la concurrence pour ses produits dentelliers rares, alors que Falcon et Achard, industriels au Puy, réclament essentiellement une protection douanière face au déferlement de produits belges très concurrentiels, et une ouverture du marché américain, principal débouché de la dentelle à la main. Lorsque le premier préconise " de soutenir, comme nous l'avons fait depuis dix ans, les écoles professionnelles pour avoir de bonnes ouvrières et continuer notre genre de fabrication " 55 , les seconds se désolent du désintérêt des jeunes gens pour l'apprentissage du métier de fabricant mais estiment que si « l'ouvrier ne veut pas d'apprentissage ", "la mère apprend le métier à sa fille ${ }^{56}$ " dans les campagnes auvergnates. Achard suggère un mode quelque peu différent de relance de l'activité dentellière, qui introduit le problème de l'innovation, grâce à l'établissement d'une fabrique modèle, qui réunirait la formation d'élèves et la création de nouveautés, destinées à " relever un peu le goût de la fabrication " en étant ensuite achetées par les fabricants ${ }^{57}$. Pour autant, Pagny, fabricant de dentelles et représentant de la Chambre consultative de Bayeux, ne voit pas dans un accroissement qualitatif la réponse à la crise des débouchés que rencontre la dentelle à la main : " la valeur artistique du peu de produits que nous fabriquons aujourd'hui est plus grande qu'autrefois ", et la production

53 Noël Jouenne, "Force et fragilité de l'industrie dentellière " in Michael Kenna (dir.), Et la dentelle ? L'industrie d'une ville : Calais, Paris, Marval, 2002.

54 Commission d'enquête sur la situation des ouvriers et des industries d'art, instituée par décret en date du 24 décembre 1881, Paris, Imprimerie A. Quantin, 1884.

55 Ibid., p. 483.

56 Ibid., p. 466.

57 Ibid., p. 464. 
mécanique a également beaucoup progressé, de sorte que la solution résiderait surtout dans l'abaissement des protections douanières à l'entrée sur les marchés de consommation américain, espagnol, russe et allemand ${ }^{58}$. À ces facteurs mécanique et douanier s'ajouterait, selon Lucien Gachon, le manque d'organisation d'une profession individuelle ${ }^{59}$.

Derrière l'apport modeste des écoles dentellières, peu productives face à la production mécanisée de masse, le volontarisme institutionnel renvoie aussi à la gestion d'un déclin. Consciemment ou non, les institutions qui soutiennent son enseignement - municipalités et chambres de commerce au premier chef - assurent le passage de la dentelle à la main de la sphère économique à la sphère artistique et patrimoniale. La dentelle continue d'animer quelques centres ruraux ; l'école dentellière remplit ainsi un rôle social, dont la reproduction d'un savoir-faire ancien et spécifique n'est que l'une des facettes. Il n'est pas indifférent que le bibliothécaire du Musée des Arts décoratifs, Léon Deshairs, écrive au passé que " pendant plusieurs siècles, cette belle industrie d'art a servi de gagne-pain à des millions de paysannes ". Les projections lumineuses que ses notices accompagnent, bien qu'elles témoignent d'une pratique pédagogique renouvelée et d'un intérêt du Musée pédagogique pour la dentelle, offrent aux institutrices des exemples anciens, à commencer par La dentellière de Vermeer ou une planche de l'Encyclopédie ${ }^{60}$.

Pourtant, l'espoir du renouveau de la dentelle à la main se perpétue dans certains cas au-delà de la Première Guerre mondiale, déjouant en partie le pronostic d'Arthur Duhem selon lequel, après la tentative scolaire, "si le succès n'était pas là, ce serait à désespérer de le trouver jamais plus ". Plusieurs prises de position révèlent la permanence du discours volontariste. Un nouveau projet de loi est déposé en 1923 par Victor Constant, député de la Haute-Loire, né au Puy-en-Velay, et affilié à la Fédération républicaine, pour protéger la fabrication de la dentelle à la main. Cette fois, les chambres de commerce sont partagées, mais quelques-unes, comme celle de Tourcoing, trouvent encore un " intérêt à la fois artistique et social à protéger la dentelle

58 Ibid., p. 426.

59 Lucien Gachon, L'Auvergne et le Velay, Paris, Gallimard, coll. "Les Provinces de France ", 4e éd. 1948, p. 220.

60 Léon Deshairs, La dentelle. Enseignement par les projections lumineuses. Notices rédigées sous le patronage de la Commission des vues instituée près du Musée pédagogique, Paris, Gustave Vitry, 1908. 
à la main "61. L'inspectrice du travail V. Paulin, en publiant son Manuel de broderies et dentelles, voit toujours en celles-ci un " complément de ressources $^{62}$ ", sans taire le renouvellement nécessaire des conditions de travail des ouvrières. Au Puy, la Première Guerre mondiale accélère la tendance générale, et crée un paysage original où les dentelles à la main et mécaniques coexistent. Après l'armistice en effet, une usine de construction de métiers s'installe, et bénéficie de la renommée des "dentelles du Puy " : l'économie de la dentelle mécanique s'insère ici dans la géographie de la dentelle à la main ${ }^{63}$, créant un équilibre nouveau entre production bon marché en ville et production de luxe dans les campagnes environnantes, là où, dans le Nord de la France, la géographie des centres de dentelle à la main reste distincte de celle des centres calaisien et caudrésien.

Certains centres isolés persévèrent, persuadés que l'enseignement de la dentelle reste un moyen privilégié de survivance de la dentelle artisanale. Cet espoir du renouveau se concrétise après la Première Guerre mondiale à Bailleul, ville dévastée par la proximité du front. Après l'armistice, l'œuvre du Retour au foyer, fondée en 1917 par la femme du député Alexandre Ribot pour aider à la reconstruction des foyers dévastés par la guerre, exprime son intérêt pour la relance de la fabrication de la dentelle ${ }^{64}$; la motivation artistique s'accompagne de la volonté d'apporter un salaire de complément aux familles. L’Américain William Nelson Cromwell, président d'honneur de cette œuvre ${ }^{65}$, agit en mécène après avoir traversé la ville en ruine en 1918 : dès 1919, le "Retour au foyer " rétablit des écoles professionnelles dans deux localités proches de Bailleul, pour accueillir une trentaine d'enfants en dehors du temps scolaire, et quelques apprenties quotidiennement. Grâce aux fonds propres de l'œuvre et à la vente de toutes les dentelles produites dans les écoles, celles-ci peuvent être

61 Annales des séances de la Chambre de commerce de Tourcoing, 12 juillet 1923, AD Nord, $77 \mathrm{~J}$ 3333.

$62 \mathrm{M}^{\text {He }} \mathrm{V}$. Paulin, Manuel de broderies et dentelles, Paris, J.-B. Baillière et Fils, "Bibliothèque professionnelle ", 1926, p. 5.

63 Comme l'observe très finement Philippe Arbos dans "L'industrie de la dentelle dans la région du Puy et son évolution actuelle ", Annales de géographie, 1923, n 178, p. 354. Il vérifie une décennie plus tard le déclin de la dentelle à la main : "L'industrie de la dentelle dans le Velay ", Annales de géographie, 1934, n² 242, p. 191-193.

64 Sur l'enseignement dentellier à Bailleul après la Première Guerre mondiale, la description la plus complète se trouve dans le catalogue d'exposition Bailleul en dentelles, Bailleul, Musée Benoît de Puydt, 1992.

65 Personnage qui fut également l'un des fondateurs du musée de la Légion d'honneur à Paris : cf. Étienne Dennery, "Hommage à William Nelson Cromwell ", Politique étrangère, 1948, vol. 13, 4, p. 303-304. 
aménagées, les enfants reçoivent nourriture et vêtements. L'enseignement de la dentelle est pérennisé grâce au soutien du rectorat : Anne-Marie Lyon, l'épouse du recteur de l'académie de Lille, Georges Lyon, s'efforce personnellement de mobiliser les énergies ${ }^{66}$. Des négociations sont engagées pour créer une école professionnelle à Bailleul. Le 9 octobre 1927, l'école dentellière de Bailleul est officiellement ouverte, installée dans un bâtiment néo-flamand construit par l'architecte Berlet. Elle est gérée par le Retour au foyer et peut accueillir plusieurs classes. Pour l'animer, une maitresse belge, Anne Comeyne, diplômée de l'École normale de dentelles de Bruges, est recrutée. En 1931, ce préapprentissage s'adresse à 35 fillettes de 7 à 11 ans, et une dizaine de jeunes filles se destinent à la profession de dentellière.

\begin{tabular}{|c|c|c|}
\hline Année & Nombre d'écoles & Nombre d'élèves \\
\hline 1860 & 8 écoles & \\
\hline 1870 & 10 écoles & 500 élèves selon Dodanthun (1905) \\
\hline 1888 & 4 écoles & Environ 200 \\
\hline \multirow[t]{2}{*}{1899} & \multirow[t]{2}{*}{3 écoles } & Moins de 100 au total pour les trois écoles \\
\hline & & $\begin{array}{l}\text { L'école de dentelle créée en } 1856 \text { par Euphrasie } \\
\text { Roelandt compte } 26 \text { élèves au } 1^{\text {er janvier } 1899 .}\end{array}$ \\
\hline 1902 & École d'E. Roelandt & Une trentaine \\
\hline 1911 & $\begin{array}{l}\text { École primaire } \\
\text { et école d'E. Roelandt }\end{array}$ & 64 élèves dans l'école d'E. Roelandt \\
\hline 1927 & École dentellière & \\
\hline 1931 & École dentellière & 35 fillettes de 7 à 11 ans, et 10 apprenties \\
\hline
\end{tabular}

Tableau $\mathrm{n}^{\circ} 3$. Aperçu de l'évolution de l'enseignement dentellier : le cas de Bailleul, $1860-1931$

L'école devient municipale en 1935, au décès de la veuve d'Alexandre Ribot ; elle dispose alors d'un effectif moyen de 80 élèves. La ville reçoit en don le bâtiment ainsi qu'une somme de 400000 francs, dont la moitié versée par William Cromwell en personne. Les dentellières de Bailleul participent aux expositions de Paris de 1933 à 1936, obtenant trois titres de " meilleures ouvrières de France ", ainsi qu'un grand prix à l'exposition internationale de Bruxelles, en

66 Lettre de $\mathrm{M}^{\mathrm{me}}$ Lyon au président de la Chambre de commerce de Lille sur la question dentellière, mai 1923, AD Nord, $76 \mathrm{~J}$ b73 d25. 
1935, puis à Paris en 1937. Des travaux de l'école dentellière de Bailleul sont également exposés à New-York en $1939^{67}$.

À Valenciennes, la renaissance de la dentelle après la guerre associe la Compagnie des chemins de fer du Nord, la municipalité et la chambre de commerce. Raoul Dautry ${ }^{68}$ et Marguerite Grange, respectivement ingénieur en chef et surintendante des œuvres sociales de la Compagnie, donnent l'impulsion. Marguerite Grange prend soin d'aller étudier l'enseignement de la dentelle à Bailleul et en Belgique, à Poperinghe et à Bruges, avant qu'un comité provisoire de patronage et de propagande ne soit formé le 20 mars 1926, permettant la création de cours aux écoles académiques et à la cité des cheminots, où est inculquée la technique du point par une ancienne élève de l'école de Bruges. Près de 80 élèves suivent ces cours, dont une vingtaine d'institutrices qui ont reçu l'appui de l'inspecteur primaire Villaume.

Une situation similaire s'observe dans l'Orne, où trois écoles d'Alençon accueillent depuis 1904 l'enseignement de la dentelle à la main grâce à des maîtresses de l'école dentellière de la chambre de commerce locale, mises gratuitement à leur disposition. Le dispositif est étendu en 1921, par un vœu de la municipalité d'Alençon appuyé par l'inspecteur d'académie, à l'école communale de la Maison d'Ozé, dans laquelle 25 à 30 des 170 élèves seraient susceptibles d'apprendre le point d'Alençon ${ }^{69}$. À nouveau, le ministère de l'Instruction publique n'est pas maître d'œuvre, mais s'associe à un effort conjoint des sphères privées et publiques ${ }^{70}$ mobilisées par la conscience d'un besoin né de la crise d'une industrie ancestrale.

Le cas de la dernière maîtresse de dentelle de Bayeux a valeur d'exemple ${ }^{71}$. En 1907, Joséphine Tranchart, âgée de 14 ans, débute son apprentissage à la Maison Lefébure, au prix d'un parcours quotidien de 6 à 8 kilomètres à pied. Devenue dentellière, Joséphine Fritteau donne des cours chaque jeudi à partir des années 1920, dans les deux écoles de la ville. Les grandes vacances sont

67 Michel Loosen, Le secret de la dentellière, Steenvoorde, Foyer culturel de l'Houtland, 1988, p. 39.

68 Personnage mieux connu grâce à la thèse de Rémi Baudouï, Raoul Dautry, 1880-1951. Le technocrate de la République, Paris, Balland, 1992.

69 Lettre de l'inspecteur d'académie au préfet de l'Orne, 5 juillet 1921, Archives départementales de l'Orne, M. 2383, reproduite dans Gérard Million, La dentelle d'Alençon (XVII - XXe siècles), Conseil général de l'Orne, Alençon, 2001, p. 117-118.

70 Arthur Malotet, La dentelle à Valenciennes, Paris, 1927, réimp. Marseille, Laffitte Reprints, 1983, p. 64-65.

71 Marie-Catherine Nobécourt, La dentelle de Bayeux, Paris, Christine Bonneton éditeur, 1982, p. 53. 
l'occasion pour quelques enfants de venir s'exercer à l'atelier de leur maîtresse. Grâce à l'un de ses mouchoirs en dentelle, elle reçoit en 1954 le diplôme de " meilleure ouvrière de France " des mains du président René Coty. Travail artistique, fruit d'un savoir-faire artisanal ancien, la production de dentelle à la main est réduite à une activité de luxe, aux vertus pédagogiques encore reconnues mais diffusées de manière quasi confidentielle.

Quelque important que soit l'espoir placé dans l'enseignement de la dentelle à la main, il serait réducteur d'y déceler la réaction univoque de l'artisanat face au développement industriel. Les industriels de la dentelle mécanique cèdent aussi, en réalité, au " courant qui nous entraîne dans les voies de l'enseignement professionnel "72, sous l'effet de la concurrence ${ }^{73}$. Un tel courant traverse l'Europe puisque, au-delà de ces quelques cas belges et français, le même phénomène pourrait être repéré de l'Irlande aux îles de la Méditerranée, en passant par l'Italie et sa tradition dentellière, perpétuée à l'école de Burano, qui prospère à partir de $1872^{74}$.

La perspective scolaire nous semble éclairer d'un jour sinon nouveau, du moins différent, l'histoire de l'industrialisation d'une production textile, aussi particulière soit-elle, et l'étude historique de l'institutionnalisation de la formation professionnelle se justifie à l'aune de ce résultat. S'opère en réalité, à travers ces survivances et expériences scolaires, une mutation de la dentelle artisanale ; source de revenus complémentaires appréciables dans les campagnes jusqu'au milieu du XIX ${ }^{\mathrm{e}}$ siècle, la dentelle, dans ce deuxième âge de la proto-industrie qui voit s'accentuer le décalage avec l'industrie mécanisée, perd lentement de son intérêt économique pour devenir une réalité patrimoniale complexe. L'insertion de la dentelle dans une véritable économie du patrimoine prend la suite, en réalité, de l'imaginaire véhiculé par celle que le fabricant de dentelle Ernest Lefébure qualifiait de " plus poétique des tissus [...] souvent

72 Jules Ferry, "L'enseignement professionnel des femmes ", discours prononcé devant l'Association philotechnique le 22 novembre 1891, reproduit dans La République des citoyens, t. II, présenté par Odile Rudelle, Paris, Imprimerie Nationale, 1996, p. 421-423.

73 Par exemple avec la création, à Calais, d'un cours de dessin et de mise en carte, par la Chambre consultative des arts et manufactures, dès 1881. Voir Henri Hénon, L'industrie des tulles et dentelles mécaniques dans le Pas-de-Calais 1815-1900, Paris, Belin Frères, 1900.

74 Exemples de ce courant européen dans Honoré Bayzelon, op. cit., qui évoque même une école fondée par une Italienne à New York, réservée aux filles d'émigrés italiens. 
chantée en vers ${ }^{75}$. La charge symbolique de la dentelle, traditionnellement associée au luxe et à l'opulence, perdure, mais n'est plus tant le support du dynamisme commercial que la nostalgie d'un art délicat. Le regain d'intérêt pour la dentelle à la Belle Époque s'avère le temps fort d'une telle mutation, tout à la fois espoir de renouveau et chant du cygne d'une activité qui a animé des territoires dentelliers durant plusieurs siècles.

Stéphane LEMBRÉ

Doctorant en histoire contemporaine, IRHiS, université Charles-de-Gaulle-Lille 3 stephane.lembre@wanadoo.fr

75 Ernest Lefébure, Broderies et dentelles, Paris, Quantin, 1887, p. 7. 